\title{
REGULARITY OF LINEAR AND POLYNOMIAL IMAGES OF SKOROHOD DIFFERENTIABLE MEASURES
}

\author{
EGOR D. KOSOV
}

\begin{abstract}
In this paper we study the regularity properties of linear and polynomial images of Skorohod differentiable measures. Firstly, we obtain estimates for the Skorohod derivative norm of a projection of a product of Scorohod differentiable measures. In the second part of the paper we prove Nikolskii-Besov regularity of a polynomial image of a Skorohod differentiable measure on $\mathbb{R}^{n}$.
\end{abstract}

Keywords: Skorohod derivative, bounded variation, Nikolskii-Besov space, Besov space, distribution of a polynomial

AMS Subject Classification: 60E05, 60E15, 60F99

\section{INTRODUCTION}

Let $\mu$ be a Skorohod differentiable probability measure on $\mathbb{R}^{n}$. In this paper we study the regularity properties of induced measures $\mu \circ f^{-1}$, where $f$ is a linear or a polynomial function.

The first part of this paper deals with measures $\mu$ of a special type: $\mu=\mu_{1} \otimes \ldots \otimes \mu_{n}$, where each $\mu_{j}$ is a probability measure on $\mathbb{R}$. Such measures are distributions of random vectors $\xi:=\left(\xi_{1}, \ldots, \xi_{n}\right)$ with independent components $\xi_{1}, \ldots, \xi_{n}$. It was proved in [18] (see also [6]) that there is an absolute constant $C$ such that, whenever $P$ is the orthogonal projection onto some $k$-dimensional subspace of $\mathbb{R}^{n}$, the density of the random vector $P \xi$ is bounded by $C^{k}$, provided that the density of each $\xi_{j}$ is not greater than 1 . In particular, in the case $k=1$ one has $C=\sqrt{2}$ as a corollary of two known results of Rogozin [19] and Ball [1]. In [16], Ball's arguments from [1] and [2] were adapted to provide some sharp bounds in the RudelsonVershynin theorem from [18]. In this paper we consider a similar question concerning bounds on the Skorohod derivative norm of the distribution of the random vector $P \xi$, provided that the distribution of each $\xi_{j}$ is Skorohod differentiable. In particular, we provide an analog of the Rudelson-Vershynin result for the norm of the Skorohod derivative (all necessary definitions will be recalled in Section 2):

For each $k \in \mathbb{N}$ there is $C(k)$ such that for any probability measures $\mu_{1}, \ldots, \mu_{n}$ on $\mathbb{R}$ with $\max _{1 \leq j \leq n}\left\|\mu_{j}^{\prime}\right\|_{\mathrm{TV}} \leq 1$ and for any orthogonal projector $P: \mathbb{R}^{n} \rightarrow \mathbb{R}^{k}$ one has

$$
\sup _{|e|=1}\left\|D_{e}\left[\mu \circ P^{-1}\right]\right\|_{\mathrm{TV}} \leq C(k),
$$

where $\mu=\mu_{1} \otimes \ldots \otimes \mu_{n}$. Moreover, $C(1)=\sqrt{2}$.

The proof is based on the recent result of Bobkov, Chistyakov and Götze [5, Lemma 4.3] about representation of any Skorohod differentiable probability measure $\nu$ on $\mathbb{R}$ as a convex mixture of uniform distributions

$$
\nu=\int \nu_{[a, b]} \pi(d a d b)
$$

such that

$$
\left\|\nu^{\prime}\right\|_{\mathrm{TV}}=\int\left\|\nu_{[a, b]}^{\prime}\right\|_{\mathrm{TV}} \pi(d a d b) .
$$

This representation enables one to reduce the proof to the case where $\xi=\left(\xi_{1}, \ldots, \xi_{n}\right)$ is uniformly distributed in the cube $Q_{n}=[-1 / 2,1 / 2]^{n}$ (i.e., $\left.\mu(d x)=I_{Q_{n}}(x) d x\right)$ and than apply 
some known results from the theory of logarithmically concave measures, since any linear image of the uniform distribution on a convex set is a logarithmically concave measure.

The second main result of this paper concerns polynomial images of general Skorohod differentiable probability measures $\mu$ and asserts that, for any polynomial $f$ of degree $d$ on $\mathbb{R}^{n}$, the distribution density of $\mu \circ f^{-1}$ belongs to the Nikolskii-Besov class $B_{1, \infty}^{1 / d}(\mathbb{R})$. The NikolskiiBesov fractional regularity of polynomial images of measures was studied in [13] and [8] in the case of Gaussian and general logarithmically concave measures. In particular, it was proved in [13] that for any log-concave measure and any polynomial $f$ of degree $d$ on $\mathbb{R}^{n}$ one has

$$
[\mathbb{D} f]^{1 / d} \int_{\mathbb{R}}\left|\rho_{f}(t+h)-\rho_{f}(t)\right| d t \leq C(d)|h|^{1 / d} \quad \forall h \in \mathbb{R},
$$

where $\rho_{f}$ is the density of the measure $\mu \circ f^{-1}$ and $\mathbb{D} f$ is the variance of $f$. In this paper we obtain a similar bound in the case where $\mu$ is a general Skorohod differentiable probability measure:

$$
\left\|B_{d}\right\|^{1 / d} \int_{\mathbb{R}}\left|\rho_{f}(t+h)-\rho_{f}(t)\right| d t \leq 24 \pi \sup _{|\theta|=1}\left\|D_{\theta} \mu\right\|_{\mathrm{TV}}|h|^{1 / d},
$$

where $f(x):=\sum_{j=0}^{d} B_{j}(x, \ldots, x)$, each $B_{j}:\left(\mathbb{R}^{n}\right)^{j} \rightarrow \mathbb{R}$ is a symmetric $j$-linear function on $\left(\mathbb{R}^{n}\right)^{j}$ and

$$
\left\|B_{d}\right\|:=\sup _{\left|x_{1}\right|=1, \ldots,\left|x_{d}\right|=1}\left|B_{d}\left(x_{1}, \ldots, x_{d}\right)\right| .
$$

The proof is based on some ideas of the papers [13] and [8] and relies on several lemmas from these papers.

\section{Preliminaries}

We first recall some definitions and notation which will be used throughout this paper.

Let $\langle\cdot, \cdot\rangle$ denote the standard inner product on $\mathbb{R}^{n}$ and let $|\cdot|$ be the usual norm generated by this inner product.

The total variation norm of a (signed) measure $\sigma$ on $\mathbb{R}^{n}$ is defined by the equality

$$
\|\sigma\|_{\mathrm{TV}}:=\sup \left\{\int \varphi d \sigma, \varphi \in C_{0}^{\infty}\left(\mathbb{R}^{n}\right),\|\varphi\|_{\infty} \leq 1\right\},
$$

where $\|\varphi\|_{\infty}:=\sup |\varphi(x)|$ and $C_{0}^{\infty}\left(\mathbb{R}^{n}\right)$ is the space of all infinitely differentiable functions with compact support.

Let $\mu$ be a Borel probability measure on $\mathbb{R}^{n}$. The measure $\mu$ is called Skorohod differentiable along $h \in \mathbb{R}^{n}$ if there is a bounded signed measure $D_{h} \mu$ such that

$$
\int_{\mathbb{R}^{n}} \partial_{h} \varphi d \mu=-\int_{\mathbb{R}^{n}} \varphi d\left(D_{h} \mu\right)
$$

for every $\varphi \in C_{0}^{\infty}\left(\mathbb{R}^{n}\right)$ (see [7]). A measure $\mu$ has the Skorohod derivatives along all vectors $h$ precisely when it possesses a density $\rho_{\mu}$ of class $B V$ (the class of functions of bounded variation). The latter means that every generalized derivative $\partial_{h} \rho_{\mu}$ is a bounded measure. In the onedimensional case in place of $D_{1} \mu$ we write $\mu^{\prime}$. For a $\mu$-measurable mapping $f: \mathbb{R}^{n} \rightarrow \mathbb{R}^{k}$, the image measure $\mu \circ f^{-1}$ is defined by the equality

for all Borel sets $A \subset \mathbb{R}^{k}$.

$$
\mu \circ f^{-1}(A):=\mu(f \in A)
$$

A Borel probability measure $\mu$ on $\mathbb{R}^{n}$ is logarithmically concave (log-concave or convex) if

$$
\mu(t A+(1-t) B) \geq \mu(A)^{t} \mu(B)^{1-t} \quad \forall t \in[0,1]
$$

for every pair of Borel sets $A, B$ (see [10]). Equivalently, $\mu$ has a density of the form $e^{-V}$ with respect to Lebesgue measure on some affine subspace $L$, where $V: L \rightarrow(-\infty,+\infty]$ is a convex 
function. Two main examples of log-concave measures are uniform distributions on convex sets and Gaussian measures. A log-concave measure $\mu$ on $\mathbb{R}^{n}$ is called isotropic if it is absolutely continuous with respect to Lebesgue measure and

$$
\int_{\mathbb{R}^{n}}\langle x, \theta\rangle \mu(d x)=0, \quad \int_{\mathbb{R}^{n}}\langle x, \theta\rangle^{2} \mu(d x)=|\theta|^{2} \quad \forall \theta \in \mathbb{R}^{n} .
$$

It is known (see [15] and also [7, Section 4.3]) that for any log-concave measure $\mu$ with a density $\rho$ and any unit vector $h$ one has

$$
\left\|D_{h} \mu\right\|_{\mathrm{TV}}=2 \int_{\langle h\rangle^{\perp}} \max _{t} \rho(x+t h) d x,
$$

where $\langle h\rangle^{\perp}$ is the orthogonal complement of $h$.

Recall that a function $f$ on $\mathbb{R}^{n}$ is a polynomial of degree $d$ if it is of the form

$$
f(x):=\sum_{j=0}^{d} B_{j}(x, \ldots, x)
$$

where each $B_{j}:\left(\mathbb{R}^{n}\right)^{j} \rightarrow \mathbb{R}$ is a symmetric $j$-linear function on $\left(\mathbb{R}^{n}\right)^{j}$ and $B_{d}$ is not identically zero. For a multilinear function $B:\left(\mathbb{R}^{n}\right)^{d} \rightarrow \mathbb{R}$ set

$$
\|B\|:=\sup _{\left|x_{1}\right|=1, \ldots,\left|x_{d}\right|=1}\left|B\left(x_{1}, \ldots, x_{d}\right)\right|,
$$

where $x_{1}, \ldots, x_{d} \in \mathbb{R}^{n}$.

For a function $g$ on $\mathbb{R}$ we set

$$
\|g\|_{p}:=\left(\int_{\mathbb{R}}|g(x)|^{p} d x\right)^{1 / p}, \quad\|g\|_{\infty}:=\sup _{x \in \mathbb{R}}|g(x)| .
$$

Recall (see [4], [17], [20], [21]) that the Nikolskii-Besov class $B_{1, \infty}^{\alpha}(\mathbb{R})$ with $\alpha \in(0,1)$ consists of all functions $g \in L^{1}(\mathbb{R})$ such that

$$
\int_{\mathbb{R}}|g(x+h)-g(x)| d x \leq C|h|^{\alpha}
$$

for some constant $C>0$.

We now formulate several known results that will be used in the proofs.

Theorem 2.1 (see [11], [3]). For every $k \in \mathbb{N}$, there is $C_{k}$ depending only on $k$ such that for every isotropic log-concave measure $\mu$ on $\mathbb{R}^{k}$ with density $\rho$ one has

$$
(\max \rho)^{1 / k} \leq C_{k} \text {. }
$$

There is an open conjecture (the so-called hyperplane conjecture) that the constant above can be chosen independent of dimension $k$. However, the best known constant so far is $C_{k} \sim k^{1 / 4}$, which is due to Klartag [11].

The following result is Corollary 2.4 in [12].

Theorem 2.2 (see [12]). For every $k \in \mathbb{N}$, there are universal constants $C, c>0$ such that, for every isotropic log-concave measure $\mu$ on $\mathbb{R}^{k}$ with density $\rho$, one has

$$
\rho(x) \leq \rho(0) e^{C k-c|x|} \quad \forall x \in \mathbb{R}^{k} .
$$

We say that a probability measure $\nu$ on $\mathbb{R}$ is represented as a convex mixture of uniform distributions with a mixing measure $\pi$ if $\pi$ is a probability measure on the half plane $\{a<b\}$ such that

$$
\int_{\mathbb{R}} \varphi d \nu=\int_{\{a<b\}} \frac{1}{b-a} \int_{a}^{b} \varphi(x) d x \pi(d a d b) \quad \forall \varphi \in C_{0}^{\infty}(\mathbb{R}) .
$$

For simplicity we denote the uniform distribution in the interval $[a, b]$ by $\nu_{[a, b]}$. 
The following theorem is Lemma 4.3 in [5]. This theorem plays a crucial role in the proof of the first main result of this paper.

Theorem 2.3 (see [5]). Any Skorohod differentiable probability measure $\nu$ on $\mathbb{R}$ can be represented as a convex mixture

$$
\nu=\int \nu_{[a, b]} \pi(d a d b)
$$

of uniform distributions with a mixing measure $\pi$ such that

$$
\left\|\nu^{\prime}\right\|_{\mathrm{TV}}=\int\left\|\nu_{[a, b]}^{\prime}\right\|_{\mathrm{TV}} \pi(d a d b)
$$

We recall that $\left\|\nu_{[a, b]}^{\prime}\right\|_{\mathrm{TV}}=\frac{2}{b-a}$.

We now present two key lemmas from [13] and [8] that will be used in the second part of the paper. The first one provides a sufficient condition for a measure on $\mathbb{R}$ to possess a density from a certain Nikolskii-Besov class (see also [9] and [14] for further development of this approach).

Lemma 2.4 (see [13], [8]). Let $\nu$ be a Borel probability measure on the real line. Assume that for every function $\varphi \in C_{0}^{\infty}(\mathbb{R})$ with $\|\varphi\|_{\infty} \leq 1$ one has

$$
\int \varphi^{\prime} d \nu \leq C\left\|\varphi^{\prime}\right\|_{\infty}^{1-\alpha}
$$

Then

$$
\left\|\nu_{h}-\nu\right\|_{\mathrm{TV}} \leq 2^{1-\alpha} C|h|^{\alpha} \quad \forall h \in \mathbb{R},
$$

where $\nu_{h}(A):=\nu(A-h)$ for all Borel sets $A \subset \mathbb{R}$. In particular, the density of the measure $\nu$ belongs to the Nikolskii-Besov class $B_{1, \infty}^{\alpha}(\mathbb{R})$.

We also need the following result.

Lemma 2.5 (see [13]). Let $\nu$ be a Borel probability measure on the real line. Assume that for every function $\varphi \in C_{0}^{\infty}(\mathbb{R})$ with $\|\varphi\|_{\infty} \leq 1$ one has

$$
\int \varphi^{\prime} d \nu \leq C\left\|\varphi^{\prime}\right\|_{\infty}^{1-\alpha}
$$

Then, for every Borel set A, one has

$$
\nu(A) \leq C \lambda(A)^{\alpha},
$$

where $\lambda$ is the standard Lebesgue measure on the real line. Moreover, if $\rho_{\nu}$ is the density of the measure $\nu$, then $\rho_{\nu} \in L^{p}(\lambda)$ whenever $1<p<\frac{1}{1-\alpha}$ and one has

$$
\left\|\rho_{\nu}\right\|_{L^{p}(\lambda)} \leq\left(p+p\left(\frac{1}{1-\alpha}-p\right)^{-1}\right)^{1 / p} C^{\frac{1}{\alpha}(1-1 / p)} .
$$

The second part of this lemma is the usual embedding theorem for Nikolskii-Besov spaces.

\section{Linear images of products of Skorohod differentiable measures}

We start with the one-dimensional case.

Theorem 3.1. Let $\mu_{1}, \ldots, \mu_{n}$ be Skorohod differentiable probability measures on $\mathbb{R}$. Then, for any linear functional $f(x)=\langle a, x\rangle$, where $a=\left(a_{1}, \ldots, a_{n}\right) \in \mathbb{R}^{n}$ with $|a|=1$, one has

$$
\left\|\left(\mu \circ f^{-1}\right)^{\prime}\right\|_{\mathrm{TV}} \leq \sqrt{2} \max _{1 \leq j \leq n}\left\|\mu_{j}^{\prime}\right\|_{\mathrm{TV}}
$$

where $\mu:=\mu_{1} \otimes \ldots \otimes \mu_{n}$. 
Proof. Applying Theorem [2.3, for an arbitrary function $\varphi \in C_{0}^{\infty}(\mathbb{R})$ with $\|\varphi\|_{\infty} \leq 1$, we have

$$
\int \varphi^{\prime}(\langle a, x\rangle) \mu(d x)=\int \frac{1}{\left(\beta_{1}-\alpha_{1}\right) \ldots\left(\beta_{n}-\alpha_{n}\right)}\left[\int_{\prod_{j=1}^{n}\left[\alpha_{j}, \beta_{j}\right]} \varphi^{\prime}(\langle a, x\rangle) d x\right] \pi_{1}\left(d \alpha_{1} d \beta_{1}\right) \ldots \pi_{n}\left(d \alpha_{n} d \beta_{n}\right) .
$$

Note that the inner integral can be represented as

$$
\int_{Q_{n}} \varphi^{\prime}(\langle L a, x\rangle+\langle a, c\rangle) d x
$$

where $Q_{n}=[-1 / 2,1 / 2]^{n}$ is the unit cube in $\mathbb{R}^{n}, \ell_{j}=\beta_{j}-\alpha_{j}, L=\operatorname{diag}\left(\ell_{1}, \ldots, \ell_{n}\right)$, and where $c=2^{-1}\left(\alpha_{1}+\beta_{1}, \ldots, \alpha_{n}+\beta_{n}\right)$. Let $\theta=|L a|^{-1} L a$, let $\psi(t)=\varphi(|L a| t+\langle a, c\rangle)$ and let $\rho_{\theta}$ be the distribution density of the random variable $\langle\theta, x\rangle$ on the probability space $\left(\mathbb{R}^{n}, \mathcal{B}\left(\mathbb{R}^{n}\right), I_{Q_{n}} d x\right)$. Then

$$
\begin{aligned}
\int_{Q_{n}} \varphi^{\prime}(\langle L a, x\rangle+\langle a, c\rangle) d x=|L a|^{-1} \int \psi^{\prime}(t) \rho_{\theta}(t) d t & \\
& \leq|L a|^{-1}\left\|\rho_{\theta}^{\prime}\right\|_{\mathrm{TV}}=|L a|^{-1} 2 \max \rho_{\theta}=2|L a|^{-1} \rho_{\theta}(0),
\end{aligned}
$$

since $\rho_{\theta}$ is even and logarithmically concave. Note that $\rho_{\theta}(0)=\left|\langle\theta\rangle^{\perp} \cap Q_{n}\right| \leq \sqrt{2}$ due to [1] (see also [2]). Thus,

$$
\int \varphi^{\prime}(\langle a, x\rangle) \mu(d x) \leq 2 \sqrt{2} \int\left(\sum_{j=1}^{n} a_{j}^{2} \ell_{j}^{2}\right)^{-1 / 2} \pi_{1}\left(d \alpha_{1} d \beta_{1}\right) \ldots \pi_{n}\left(d \alpha_{n} d \beta_{n}\right),
$$

where $\ell_{j}=\beta_{j}-\alpha_{j}$. By the convexity of the function $t \rightarrow t^{-1 / 2}$ for $t>0$ we have

$$
\left(\sum_{j=1}^{n} a_{j}^{2} \ell_{j}^{2}\right)^{-1 / 2} \leq \sum_{j=1}^{n} a_{j}^{2} \ell_{j}^{-1}
$$

We also recall that $\left\|\nu_{\left[\alpha_{j}, \beta_{j}\right]}^{\prime}\right\|_{\mathrm{TV}}=2 \ell_{j}^{-1}$, where $\nu_{\left[\alpha_{j}, \beta_{j}\right]}$ is the uniform distribution on the interval $\left[\alpha_{j}, \beta_{j}\right]$, which implies that

$$
\begin{aligned}
\int \varphi^{\prime}(\langle a, x\rangle) \mu(d x) \leq \sqrt{2} \int \sum_{j=1}^{n} a_{j}^{2}\left\|\nu_{\left[\alpha_{j}, \beta_{j}\right]}^{\prime}\right\|_{\mathrm{TV}} \pi_{1}\left(d \alpha_{1} d \beta_{1}\right) \ldots \pi_{n}\left(d \alpha_{n} d \beta_{n}\right) & \\
& =\sqrt{2} \sum_{j=1}^{n} a_{j}^{2}\left\|\mu_{j}^{\prime}\right\|_{\mathrm{TV}} \leq \sqrt{2} \max _{1 \leq j \leq n}\left\|\mu_{j}^{\prime}\right\|_{\mathrm{TV}} .
\end{aligned}
$$

The theorem is proved.

We now proceed to the multidimensional setting.

Theorem 3.2. Let $k \in \mathbb{N}$. Then there is $C(k)$, depending only on $k$, such that, for any Skorohod differentiable probability measures $\mu_{1}, \ldots, \mu_{n}$ on $\mathbb{R}$ and for any mapping $F: \mathbb{R}^{n} \rightarrow \mathbb{R}^{k}$ of the form $F(x)=A x$, where $A$ is a $k \times n$ matrix with orthonormal rows $a_{1}, \ldots, a_{k}$, one has

$$
\left\|\partial_{e}\left(\mu \circ F^{-1}\right)\right\|_{\mathrm{TV}} \leq C(k) \max _{1 \leq j \leq n}\left\|\mu_{j}^{\prime}\right\|_{\mathrm{TV}} \quad \forall e \in \mathbb{R}^{k},|e|=1,
$$

where $\mu:=\mu_{1} \otimes \ldots \otimes \mu_{n}$.

Proof. Applying Theorem 2.3, for any function $\varphi \in C_{0}^{\infty}\left(\mathbb{R}^{k}\right)$ with $\|\varphi\|_{\infty} \leq 1$ and any unit vector $e$, one has

$$
\int \partial_{e} \varphi(A x) \mu(d x)=\int \frac{1}{\left(\beta_{1}-\alpha_{1}\right) \ldots\left(\beta_{n}-\alpha_{n}\right)}\left[\int_{\prod_{j=1}^{n}\left[\alpha_{j}, \beta_{j}\right]} \partial_{e} \varphi(A x) d x\right] \pi_{1}\left(d \alpha_{1} d \beta_{1}\right) \ldots \pi_{n}\left(d \alpha_{n} d \beta_{n}\right) .
$$


The inner integral is equal to

$$
\int_{Q_{n}} \partial_{e} \varphi(A L x+A c) d x
$$

where $Q_{n}=[-1 / 2,1 / 2]^{n}$ is the unit cube in $\mathbb{R}^{n}, L=\operatorname{diag}\left(\ell_{1}, \ldots, \ell_{n}\right), \ell_{j}=\beta_{j}-\alpha_{j}$, and $c=$ $\left(\left(\alpha_{1}+\beta_{1}\right) / 2, \ldots,\left(\alpha_{n}+\beta_{n}\right) / 2\right)$. Let $\sigma$ be the distribution of the random vector $\left(A L^{2} A^{T}\right)^{-1 / 2} A L x$ on the probability space $\left(\mathbb{R}^{n}, \mathcal{B}\left(\mathbb{R}^{n}\right), I_{Q_{n}} d x\right)$. Note that $\sigma$ is log-concave and isotropic. Indeed, letting $B=A L$, for any $\theta \in \mathbb{R}^{k}$ we have

$$
\begin{aligned}
\int\langle y, \theta\rangle^{2} \sigma(d y) & =\int_{Q_{n}}\left\langle\left(B B^{T}\right)^{-1 / 2} B x, \theta\right\rangle^{2} d x=\int_{Q_{n}}\left\langle x, B^{T}\left(B B^{T}\right)^{-1 / 2} \theta\right\rangle^{2} d x \\
= & \left\langle B^{T}\left(B B^{T}\right)^{-1 / 2} \theta, B^{T}\left(B B^{T}\right)^{-1 / 2} \theta\right\rangle=\left\langle\left(B B^{T}\right)^{-1 / 2} B B^{T}\left(B B^{T}\right)^{-1 / 2} \theta, \theta\right\rangle=|\theta|^{2} .
\end{aligned}
$$

Let $\rho$ be the density of the measure $\sigma$. By Theorems 2.1 and 2.2 there is $C_{k}$, depending only on $k$, and there are two absolute constants $C$ and $c$ such that

$$
\rho(x) \leq C_{k}^{k} e^{C k-c|x|}
$$

Thus, for every unit vector $h$

$$
\left\|D_{h} \sigma\right\|_{\mathrm{TV}}=2 \int_{\langle h\rangle^{\perp}} \max _{t} \rho(x+t h) d x \leq 2 C_{k}^{k} e^{C k} \int_{\mathbb{R}^{k-1}} e^{-c \sqrt{x_{1}^{2}+\ldots+x_{k-1}^{2}}} d x_{1} \ldots d x_{k-1}=C_{1}(k) .
$$

We now consider the function

$$
\psi(x):=\varphi\left(\left(A L^{2} A^{T}\right)^{1 / 2} x+A c\right)
$$

and the vector

$$
\theta:=\left|\left(A L^{2} A^{T}\right)^{-1 / 2} e\right|^{-1}\left(A L^{2} A^{T}\right)^{-1 / 2} e .
$$

Note that

$$
\begin{aligned}
\int \partial_{\theta} \psi d \sigma=\left|\left(A L^{2} A^{T}\right)^{-1 / 2} e\right|^{-1} \int\left(\partial_{e} \varphi\right)\left(\left(A L^{2} A^{T}\right)^{1 / 2} y+A c\right) \sigma(d y) & \\
& =\left|\left(A L^{2} A^{T}\right)^{-1 / 2} e\right|^{-1} \int_{Q_{n}} \partial_{e} \varphi(A L x+A c) d x
\end{aligned}
$$

which implies the bound

$$
\int_{Q_{n}} \partial_{e} \varphi(A L x+A c) d x \leq C_{1}(k)\left|\left(A L^{2} A^{T}\right)^{-1 / 2} e\right| \leq C_{1}(k)\left\|\left(A L^{2} A^{T}\right)^{-1 / 2}\right\|_{o p},
$$

where for any matrix $B$ its operator norm is $\|B\|_{o p}:=\sup _{|h|=1}|B h|$. Let us now recall that

$$
\left\|\left(A L^{2} A^{T}\right)^{-1 / 2}\right\|_{o p}=\left(\inf _{|h|=1}\left\langle\left(A L^{2} A^{T}\right)^{1 / 2} h,\left(A L^{2} A^{T}\right)^{1 / 2} h\right\rangle\right)^{-1 / 2}=\sup _{|h|=1}\left(\left\langle A L^{2} A^{T} h, h\right\rangle\right)^{-1 / 2} .
$$

We note that the function $R_{h}\left(\ell_{1}^{2}, \ldots, \ell_{n}^{2}\right)=\left\langle A L^{2} A^{T} h, h\right\rangle$ is linear for any fixed unit vector $h$. Thus,

$$
R_{h}\left(\ell_{1}^{2}, \ldots, \ell_{n}^{2}\right)=k_{1}(h) \ell_{1}^{2}+\ldots+k_{n}(h) \ell_{n}^{2}
$$

Moreover,

$$
k_{1}(h)+\ldots+k_{n}(h)=R_{h}(1, \ldots, 1)=\left\langle A^{T} h, A^{T} h\right\rangle=1
$$

and

$$
k_{j}(h)=R_{h}(0, \ldots, 1, \ldots, 0) \geq 0 .
$$


By the convexity of the function $t \mapsto t^{-1 / 2}$ for $t>0$ we get

$$
\begin{aligned}
\left\|\left(A L^{2} A^{T}\right)^{-1 / 2}\right\|_{o p}=\sup _{|h|=1}\left(k_{1}(h) \ell_{1}^{2}+\ldots+k_{n}(h) \ell_{n}^{2}\right)^{-1 / 2} & \\
\leq \sup _{|h|=1}\left[k_{1}(h) \ell_{1}^{-1}+\ldots+k_{n}(h) \ell_{n}^{-1}\right]= & \sup _{|h|=1} R_{h}\left(\ell_{1}^{-1}, \ldots, \ell_{n}^{-1}\right)=\sup _{|h|=1}\left\langle A L^{-1} A^{T} h, h\right\rangle \\
& \leq \operatorname{tr}\left[A L^{-1} A^{T}\right]=\operatorname{tr}\left[A^{T} A L^{-1}\right]=\sum_{j=1}^{n}\left(\sum_{i=1}^{k} a_{j, i}^{2}\right) \ell_{j}^{-1} .
\end{aligned}
$$

Recall that $\left\|\nu_{\left[\alpha_{j}, \beta_{j}\right]}^{\prime}\right\|_{\mathrm{TV}}=2 \ell_{j}^{-1}$. Hence

$$
\begin{array}{r}
\int \partial_{e} \varphi(A x) \mu(d x) \leq 2^{-1} C_{1}(k) \int \sum_{j=1}^{n}\left(\sum_{i=1}^{k}\left(a_{j, i}\right)^{2}\right)\left\|\nu_{\left[\alpha_{j}, \beta_{j}\right]}^{\prime}\right\|_{\mathrm{TV}} \pi_{1}\left(d \alpha_{1} d \beta_{1}\right) \ldots \pi_{n}\left(d \alpha_{n} d \beta_{n}\right) \\
=C_{1}(k) 2^{-1} \sum_{j=1}^{n}\left(\sum_{i=1}^{k}\left(a_{j, i}\right)^{2}\right)\left\|\mu_{j}^{\prime}\right\|_{\mathrm{TV}} \leq 2^{-1} k C_{1}(k) \max _{1 \leq j \leq n}\left\|\mu_{j}^{\prime}\right\|_{\mathrm{TV}}
\end{array}
$$

which completes the proof.

Remark 3.3. We note that the constant $C(k)$ obtained in the previous theorem equals

$$
k C_{k}^{k} e^{C k} \int_{\mathbb{R}^{k-1}} e^{-c \sqrt{x_{1}^{2}+\ldots+x_{k-1}^{2}}} d x_{1} \ldots d x_{k-1},
$$

where $C_{k} \sim k^{1 / 4}$. The integral above is equal to

$$
\frac{(k-1) \pi^{\frac{k-1}{2}} \Gamma(k-1)}{c^{k-1} \Gamma\left(\frac{k-1}{2}+1\right)} .
$$

Thus, $C(k) \sim(C k)^{\frac{3 k}{4}}$. The hyperplane conjecture asserts that $C_{k}$ is actually independent of $k$ and then $C(k)$ must be equivalent to $(C k)^{k / 2}$. It is interesting to understand the actual dependence of $C(k)$ of $k$.

\section{Polynomial images of General Skorohod differentiable measures}

In this section we study the regularity properties of polynomial images of Skorohod differentiable measures on $\mathbb{R}^{n}$.

We start with the following technical lemma.

Lemma 4.1. Let $\mu$ be a probability measure on $\mathbb{R}^{n}$ Skorohod differentiable along a unit vector $\theta \in \mathbb{R}^{n}$ and let $f$ be a polynomial of degree $d$ on $\mathbb{R}^{n}$. Consider the function

$$
F:=\frac{f}{f^{2}+a^{2}} .
$$

Let $g \in C^{\infty}\left(\mathbb{R}^{n}\right) \cap L^{\infty}\left(\mathbb{R}^{n}\right)$ be a function such that $\partial_{\theta} g F \in L^{1}(\mu)$. Then

$$
\int \partial_{\theta} g F d \mu \leq(3(d+1) \pi+1 / 2)\left\|D_{\theta} \mu\right\|_{\mathrm{TV}} a^{-1}\|g\|_{\infty}
$$

Proof. Without loss of generality we can assume that $a=1$. For every function $\psi \in C_{0}^{\infty}\left(\mathbb{R}^{n}\right)$ we have

$$
\int \partial_{\theta} \psi F d \mu=-\int \psi\left[\frac{\partial_{\theta} f}{f^{2}+1}-\frac{2 f^{2} \partial_{\theta} f}{\left(f^{2}+1\right)^{2}}\right] d \mu-\int \psi(x) F(x) D_{\theta} \mu(d x) .
$$

The right-hand side is estimated from above by

$$
\|\psi\|_{\infty}\left(3 \int \frac{\left|\partial_{\theta} f\right|}{f^{2}+1} d \mu_{n}+2^{-1}\left\|D_{\theta} \mu\right\|_{\mathrm{TV}}\right) .
$$


Let us now note that

$$
\begin{aligned}
& \int \frac{\left|\partial_{\theta} f\right|}{f^{2}+1} d \mu=\int_{\langle\theta\rangle \perp} \int_{\mathbb{R}}\left|\frac{d}{d t} \operatorname{arctg}(f(y+t \theta))\right| \rho(y+t \theta) d t d y \\
& \leq(d+1) \pi \int_{\langle\theta\rangle \perp} \sup _{\tau} \rho(y+\tau \theta) d y \leq(d+1) \pi\left\|D_{\theta} \mu\right\|_{\mathrm{TV}} .
\end{aligned}
$$

Thus,

$$
\int \partial_{\theta} \psi F d \mu \leq(3(d+1) \pi+1 / 2)\left\|D_{\theta} \mu\right\|_{\mathrm{TV}}\|\psi\|_{\infty}
$$

Let $\psi_{k}(x)=\psi(x / k)$, where $\psi \in C_{0}^{\infty}\left(\mathbb{R}^{n}\right)$ is a function such that $\psi(x)=1$ for $|x| \leq 1, \psi(x)=0$ for $|x|>2$, and $\psi(x) \in[0,1]$ for each $x$. We now apply estimate (11) to the function $\psi_{k} g$ :

$$
\int \partial_{\theta}\left(\psi_{k} g\right) F d \mu \leq(3(d+1) \pi+1 / 2)\left\|D_{\theta} \mu\right\|_{\mathrm{TV}}\|g\|_{\infty}
$$

Note that

$$
\int \partial_{\theta}\left(\psi_{k} g\right) F d \mu=\int g \partial_{\theta} \psi_{k} F d \mu+\int \psi_{k} \partial_{\theta} g F d \mu
$$

where the first term tends to zero and the second term tends to $\int \partial_{\theta} g F d \mu$. Thus, we have obtained the desired estimate. The lemma is proved.

Corollary 4.2. Let $\mu$ be a probability measure on $\mathbb{R}^{n}$ Skorohod differentiable along a unit vector $\theta \in \mathbb{R}^{n}$ and let $f$ be a polynomial of degree $d$ on $\mathbb{R}^{n}$. Then, for any function $\varphi \in C_{b}^{\infty}(\mathbb{R})$ with $\|\varphi\|_{\infty} \leq 1$, any unit vector $\theta$, and any positive number $\varepsilon$, one has

$$
\int \varphi^{\prime}(f) \partial_{\theta} f \frac{\partial_{\theta} f}{\left(\partial_{\theta} f\right)^{2}+\varepsilon} d \mu_{n} \leq(3 \pi d+1 / 2)\left\|D_{\theta} \mu\right\|_{\mathrm{TV}} \varepsilon^{-1 / 2}
$$

We are now ready to prove our second main result.

Theorem 4.3. Let $\mu$ be a probability measure on $\mathbb{R}^{n}$ Skorohod differentiable along every vector $h \in \mathbb{R}^{n}$ and let $f$ be a polynomial of degree $d$ on $\mathbb{R}^{n}$ of the form $f(x)=\sum_{j=0}^{d} B_{j}(x, \ldots, x)$, where each $B_{j}:\left(\mathbb{R}^{n}\right)^{j} \rightarrow \mathbb{R}$ is a $j$-linear symmetric function. Then, for an arbitrary function $\varphi \in C_{b}^{\infty}(\mathbb{R})$ with $\|\varphi\|_{\infty} \leq 1$, one has

$$
\int \varphi^{\prime}(f) d \mu \leq 12 \pi \sup _{|\theta|=1}\left\|D_{\theta} \mu\right\|_{\mathrm{TV}}\left\|B_{d}\right\|^{-1 / d}\left\|\varphi^{\prime}\right\|_{\infty}^{1-1 / d}
$$

and the measure $\mu \circ f^{-1}$ possess a density from the Nikolskii-Besov class $B_{1, \infty}^{1 / d}(\mathbb{R})$. Moreover,

$$
\mu(f \in A) \leq 12 \pi \sup _{|\theta|=1}\left\|D_{\theta} \mu\right\|_{\mathrm{TV}}\left\|B_{d}\right\|^{-1 / d}[\lambda(A)]^{1 / d} .
$$

Proof. We will prove this theorem by induction on $d$.

The base of induction. We first consider the case $d=1$. In this case $f(x)=\langle a, x\rangle+b=$ $\sum_{k=1}^{n} a_{k} x_{k}+b$ for some $a \in \mathbb{R}^{n}$ and $b \in \mathbb{R}$. Note that for an arbitrary function $\varphi \in C_{0}^{\infty}(\mathbb{R})$ with $\|\varphi\|_{\infty} \leq 1$ one has

$$
\int \varphi^{\prime}(f) d \mu=-|a|^{-2} \int \varphi\left(\sum_{k=1}^{n} a_{k} x_{k}+b\right) D_{a} \mu(d x) \leq|a|^{-2}\left\|D_{a} \mu\right\|_{\mathrm{TV}} \leq|a|^{-1} \sup _{|\theta|=1}\left\|D_{\theta} \mu\right\|_{\mathrm{TV}} .
$$

The induction step. Assume that estimate (2) is valid for every polynomial of degree not greater than $d$ and let $f$ be a polynomial of degree $d+1$. Note that for an arbitrary function $\varphi \in C_{0}^{\infty}(\mathbb{R})$ with $\|\varphi\|_{\infty} \leq 1$ we have

$$
\int \varphi^{\prime}(f) d \mu=\int \varphi^{\prime}(f) \partial_{\theta} f \frac{\partial_{\theta} f}{\left(\partial_{\theta} f\right)^{2}+\varepsilon} d \mu+\varepsilon \int \varphi^{\prime}(f(x))\left(\left(\partial_{\theta} f\right)^{2}+\varepsilon\right)^{-1} d \mu
$$


for an arbitrary number $\varepsilon>0$ and for an arbitrary unit vector $\theta \in \mathbb{R}^{n}$. Let us estimate each term separately. For the first term by Corollary 4.2 we have

$$
\int \varphi^{\prime}(f) \partial_{\theta} f \frac{\partial_{\theta} f}{\left(\partial_{\theta} f\right)^{2}+\varepsilon} d \mu_{n} \leq(3 \pi d+1 / 2) \sup _{|h|=1}\left\|D_{h} \mu\right\|_{\mathrm{TV}} \varepsilon^{-1 / 2}
$$

By the equality $\partial_{\theta} f(x)=\sum_{j=1}^{d+1} j B_{j}(x, \ldots, x, \theta)$, the induction hypothesis, and Lemma 2.5, the second term in (3) can be estimated as follows:

$$
\begin{aligned}
& \varepsilon \int \varphi^{\prime}(f)\left(\left(\partial_{\theta} f\right)^{2}+\varepsilon\right)^{-1} d \mu \leq \varepsilon\left\|\varphi^{\prime}\right\|_{\infty} \int_{0}^{1 / \varepsilon} \mu\left(\left(\partial_{\theta} f\right)^{2} \leq 1 / t-\varepsilon\right) d t \\
& =\varepsilon\left\|\varphi^{\prime}\right\|_{\infty} \int_{0}^{\infty}(s+\varepsilon)^{-2} \mu\left(\left|\partial_{\theta} f\right| \leq \sqrt{s}\right) d s \\
& \leq \varepsilon\left\|\varphi^{\prime}\right\|_{\infty} 12 \pi \sup _{|h|=1}\left\|D_{h} \mu\right\|_{\mathrm{TV}}(d+1)^{-1 / d}\left\|B_{d+1}(\cdot, \ldots, \cdot, \theta)\right\|^{-1 / d} 2^{1 / d} \int_{0}^{\infty}(s+\varepsilon)^{-2} s^{1 / 2 d} d s \\
& =\varepsilon^{1 / 2 d}\left\|\varphi^{\prime}\right\|_{\infty} \sup _{|h|=1}\left\|D_{h} \mu\right\|_{\mathrm{TV}}\left\|B_{d+1}(\cdot, \ldots, \cdot, \theta)\right\|^{-1 / d} 2^{1 / d}(d+1)^{-1 / d} 12 \pi \int_{0}^{\infty}(s+1)^{-2} s^{1 / 2 d} d s .
\end{aligned}
$$

Thus, we have

$$
\begin{aligned}
& \int \varphi^{\prime}(f) d \mu \leq \varepsilon^{-1 / 2}(3 \pi d+1 / 2) \sup _{|h|=1}\left\|D_{h} \mu\right\|_{\mathrm{TV}} \\
& +\varepsilon^{1 / 2 d}\left\|\varphi^{\prime}\right\|_{\infty} \sup _{|h|=1}\left\|D_{h} \mu\right\|_{\mathrm{TV}}\left\|B_{d+1}(\cdot, \ldots, \cdot, \theta)\right\|^{-1 / d} 2^{1 / d}(d+1)^{-1 / d} 12 \pi \int_{0}^{\infty}(s+1)^{-2} s^{1 / 2 d} d s .
\end{aligned}
$$

Setting

$$
\varepsilon=(3 \pi d+1 / 2)^{\frac{2 d}{d+1}}\left(\left\|\varphi^{\prime}\right\|_{\infty}\left\|B_{d+1}(\cdot, \ldots, \cdot, \theta)\right\|^{-\frac{1}{d}} 2^{\frac{1}{d}}(d+1)^{-\frac{1}{d}} 12 \pi \int_{0}^{\infty}(s+1)^{-2} s^{\frac{1}{2 d}} d s\right)^{-\frac{2 d}{d+1}},
$$

we get

$$
\int \varphi^{\prime}(f) d \mu \leq c_{d+1} \sup _{|h|=1}\left\|D_{h} \mu\right\|_{\mathrm{TV}}\left\|B_{d+1}(\cdot, \ldots, \cdot, \theta)\right\|^{-1 /(d+1)}\left\|\varphi^{\prime}\right\|_{\infty}^{1-1 /(d+1)}
$$

with

$$
c_{d+1}=(3 \pi d+1 / 2)^{\frac{1}{d+1}}\left(2^{1 / d}(d+1)^{-1 / d} 12 \pi \int_{0}^{\infty}(s+1)^{-2} s^{1 / 2 d} d s\right)^{\frac{d}{d+1}} .
$$

Note that

$$
\int_{0}^{\infty}(s+1)^{-2} s^{1 / 2 d} d s \leq\left(\int_{0}^{\infty}(s+1)^{-2} s^{1 / 2} d s\right)^{1 / d}=(\pi / 2)^{1 / d} \leq 2^{1 / d}
$$

and also note that

$$
\frac{3 \pi d+1 / 2}{d+1} \leq 3 \pi
$$

Thus, $c_{d+1} \leq 12 \pi$ and taking the infimum over $\theta$ we get the desired bound. Theorem is proved.

From Lemma 2.5 we get the following result on the integrability of the distribution density.

Corollary 4.4. Let $\mu$ be a measure on $\mathbb{R}^{n}$ Skorohod differentiable along every vector $h \in \mathbb{R}^{n}$ and let $f$ be a polynomial of degree $d$ on $\mathbb{R}^{n}$ of the form $f(x)=\sum_{j=0}^{d} B_{j}(x, \ldots, x)$, where each $B_{j}:\left(\mathbb{R}^{n}\right)^{j} \rightarrow \mathbb{R}$ is a $j$-linear symmetric function. Then the density $\rho_{f}$ of the measure $\mu \circ f^{-1}$ is integrable to every power $p \in\left[1, \frac{d}{d-1}\right)$ and

$$
\left\|\rho_{f}\right\|_{p} \leq c(p, d)\left(\sup _{|\theta|=1}\left\|D_{\theta} \mu\right\|_{\mathrm{TV}}\right)^{d(1-1 / p)}\left\|B_{d}\right\|^{1 / p-1}
$$


where $c(p, d)=\left(p+p\left(\frac{d}{d-1}-p\right)^{-1}\right)^{1 / p}(12 \pi)^{d(1-1 / p)}$.

From [13, Lemma 2.3] (see also [8, Theorem 3.2]) we get the following corollary.

Corollary 4.5. Let $\mu$ be a measure on $\mathbb{R}^{n}$ Skorohod differentiable along every vector $h \in \mathbb{R}^{n}$ and let $f_{1}$ and $f_{2}$ be two polynomials of degree $d$ on $\mathbb{R}^{n}$ of the form $f_{i}(x)=\sum_{j=0}^{d} B_{j}^{i}(x, \ldots, x)$, where each $B_{j}^{i}:\left(\mathbb{R}^{n}\right)^{j} \rightarrow \mathbb{R}$ is a $j$-linear symmetric function, $i=1,2$. Then

$$
\left\|\mu \circ f_{1}^{-1}-\mu \circ f_{2}^{-1}\right\|_{\mathrm{TV}} \leq C\left\|\mu \circ f_{1}^{-1}-\mu \circ f_{2}^{-1}\right\|_{\mathrm{KR}}^{1 /(1+d)},
$$

where

$$
C=2\left(1+12 \pi \sup _{|\theta|=1}\left\|D_{\theta} \mu\right\|_{\mathrm{TV}}\left(\left\|B_{d}^{1}\right\|^{-1 / d}+\left\|B_{d}^{2}\right\|^{-1 / d}\right)\right)
$$

and $\|\cdot\|_{\mathrm{KR}}$ is the Kantorovich-Rubinstein norm of a measure defined by

$$
\|\nu\|_{\mathrm{KR}}:=\sup \left\{\int \varphi d \nu, \varphi \in C_{0}^{\infty}\left(\mathbb{R}^{n}\right),\|\varphi\|_{\infty} \leq 1,\left\|\varphi^{\prime}\right\|_{\infty} \leq 1\right\} .
$$

The author is a Young Russian Mathematics award winner and would like to thank its sponsors and jury.

The article was prepared within the framework of the HSE University Basic Research Program and funded by the Russian Academic Excellence Project '5-100'.

This research was supported by the RFBR Grant 17-01-00662 and by the Foundation for the Advancement of Theoretical Physics and Mathematics "BASIS".

\section{REFERENCES}

[1] Ball, K.: Cube slicing in $\mathbb{R}^{n}$. Proceedings of the AMS, 97(3), 465-473 (1986)

[2] Ball, K.: Volumes of sections of cubes and related problems. In: Geometric aspects of functional analysis, pp. 251-260 (1989)

[3] Ball, K.: Logarithmically concave functions and sections of convex sets in $\mathbb{R}^{n}$. Studia Math. 88(1), 69-84 (1988)

[4] Besov, O.V., Il'in, V.P., Nikolskiu, S.M.: Integral representations of functions and imbedding theorems. V. I, II. Winston \& Sons, Washington; Halsted Press, New York - Toronto - London $(1978,1979)$

[5] Bobkov, S.G., Chistyakov, G.P., Götze, F.: Fisher information and the central limit theorem. Probab. Theory Related Fields. 159(1-2), 1-59 (2014)

[6] Bobkov, S.G. , Chistyakov, G.P.: Bounds on the maximum of the density for sums of independent random variables, J. Math. Sci. 199(2), 100-106 (2014)

[7] Bogachev, V.I.: Differentiable measures and the Malliavin calculus. Amer. Math. Soc., Providence, Rhode Island (2010)

[8] Bogachev, V.I., Kosov, E.D., Zelenov, G.I.: Fractional smoothness of distributions of polynomials and a fractional analog of the Hardy-Landau-Littlewood inequality. Amer. Math. Soc. 370(6), 4401-4432 (2018)

[9] Bogachev, V.I., Kosov, E.D., Popova, S.N.: A new approach to Nikolskii-Besov classes. to appear in Mosc. Math. J.

[10] Borell, C.: Convex measures on locally convex spaces. Ark. Math. 12, 239-252 (1974)

[11] Klartag, B.: On convex perturbations with a bounded isotropic constant. Geom. Funct. Anal. 16(6), 12741290 (2006)

[12] Klartag, B.: Power-law estimates for the central limit theorem for convex sets. J. Funct. Anal. 245(1), 284-310 (2007)

[13] Kosov, E.D.: Fractional smoothness of images of logarithmically concave measures under polynomials. J. Math. Anal. Appl. 462(1), 390-406 (2018)

[14] Kosov, E.D.: Besov classes on finite and infinite dimensional spaces, to appear in Sbornik Math.

[15] Krugova, E. P.: On translates of convex measures. Sbornik Math. 188(2), 227-236 (1997)

[16] Livshyts, G., Paouris, G., Pivovarov, P.: On sharp bounds for marginal densities of product measures. Israel J. Math. 216(2), 877-889 (2016)

[17] Nikolskii, S.M.: Approximation of functions of several variables and imbedding theorems. Transl. from the Russian. Springer-Verlag, New York - Heidelberg (1975) (Russian ed.: Moscow, 1977). 
[18] Rudelson, M., Vershynin, R.: Small ball probabilities for linear images of high-dimensional distributions. Int. Math. Res. Not. 2015(19), 9594-9617 (2014)

[19] Rogozin, B.A.: The estimate of the maximum of the convolution of bounded densities. Teor. Veroyatn. Primen. 32(1), 53-61 (1987)

[20] Triebel, H.: Theory of function spaces, II, Birkhäuser Verlag, Basel (1992)

[21] Stein, E.: Singular integrals and differentiability properties of functions. Princeton University Press, Princeton $(1970)$ 